\title{
Bitcoin and Market-(In)Efficiency: a Systematic Time Series Approach
}

\author{
Nils Bundi • Marc Wildi
}

Received: date / Accepted: date

\begin{abstract}
Recently, cryptocurrencies have received substantial attention by investors given their innovative features, simplicity and transparency. We here analyse the increasingly popular Bitcoin and verify pertinence of the efficient market hypothesis. Recent research suggests that Bitcoin markets, while inefficient in their early days, transitioned into efficient markets recently. We challenge this claim by proposing simple trading strategies based on moving average filters, on classic time series models as well as on non-linear neural nets. Our findings suggest that trading performances of our designs are significantly positive; moreover, linear and non-linear approaches perform similarly except at singular time periods of the Bitcoin; finally, our results suggest that markets are becoming less rather than more efficient towards the sample end of the data.
\end{abstract}

Keywords Bitcoin - Cryptocurrencies · Efficient Market Hypothesis · Time Series Analysis · Moving Average Filters

\footnotetext{
Nils Bundi

School of Business, Stevens Institute of Technology

1 Castle Point Terrace, Hoboken, NJ 07030

USA

E-mail: nbundi@stevens.edu

Marc Wildi

School of Engineering, Zurich University of Applied Sciences

Technikumstrasse 9,8400 Winterthur

Switzerland

E-mail: marc.wildi@zhaw.ch
} 


\section{Introduction}

Recently, cryptocurrencies have received substantial attention by investors given their innovative features, simplicity, transparency and their increasing popularity. In the period from February 2016 to March 2017, the market capitalization of cryptocurrencies has tripled, reaching a level of about 27 billion USD (Hileman and Rauchs, 2017) whereby the lion's share of roughly $70 \%$ falls upon the Bitcoin. The number of Bitcoin wallet owners is estimated to have quadrupled from about 1 million in 2013 to about 4 million in 2017 and the average daily number of trades has approached 300,000. Likewise, the price dynamics of Bitcoin show evidence of strong volatility and heavy tail behavior which seem to differ fundamentally from classic currencies, see for example Baur et al. (2018); Osterrieder and Lorenz (2017); Carrick (2016); Kasper (2017) and Osterrieder (2017); Chan et al. (2017). To some extent these peculiarities can be attributed to market liquidity which cannot compete with classic securities exchanges and which varies across Bitcoin exchanges, see (Loi, 2017). Nonetheless, Bitcoin is increasingly perceived as a viable alternative investment opportunity with potential for portfolio diversification and risk hedging, see for example Chuen et al. (2017); Brière et al. (2015); Dyhrberg (2016); Petukhina et al. (2018).

In this context, given the rising importance of Bitcoin as well as its nonstandard price dynamics, we propose to verify pertinence of the so-called efficient market hypothesis (EMH), as proposed by Fama (1970). In essence, the EMH postulates that efficient markets reflect all past information (weakform), public information (semi-strong form), or public and private information (strong form) in market prices. Verification of the EMH is important for market particpants as it implies that such information cannot be used to make persistent profits on trading on the market. In summary, recent research on the topic is inconclusive as to whether Bitcoin markets are efficient under the $\mathrm{EMH}$ or not. Some findings suggest that Bitcoin markets, while inefficient in their early days, transitioned into efficient markets recently. Others find support for the adaptive market hypothesis (AMH), an alternative theory that builds on evolutionary principles and assumes markets and market efficiency evolve over time.

We here add to this growing body of literature by analyzing effective as opposed to theoretical market efficiency. While previous work focused on the latter approach by verifying theoretical features of Bitcoin prices in efficient markets we here evaluate whether such features could, in fact, be consistently exploited for economic profit. Therefore, we test performance of various trading strategies which under the weak form of market efficiency should be not statistically different from zero or even negative when accounting for trading cost.

After a review of related literature and a presentation of the used data, we start our analysis by unfolding serial dependence in log-returns of Bitcoin. Based on these findings, we then challenge the previous body of research by applying simple trading strategies based on moving average filters, on classic 
time series models as well as on non-linear neural nets. We account for costs by crossing the bid-ask spread at each trade so that positive net performances would be indicative of effective market inefficiency. Our results reveal that net performances are positive for all proposed strategies; moreover, positive performances are statistically significant for almost all designs; also, comparisons of linear and non-linear approaches suggest that non-linearities of Bitcoin are confined to relatively short 'abnormal' time episodes around the breakdown of the currency in early 2018; finally, the performances improve towards the sample end suggesting that markets are becoming more, not less, inefficient.

\section{Related Literature}

Fama (1970) suggested three categories of informational efficiency: (i) weak efficiency, if current prices reflect the information contained in past prices, (ii) semi-strong efficiency, if prices reflect all public information and (iii) strong efficiency, if prices reflect all public and private information. A large body of literature has formed around the verification of the EMH in stock markets, commodities markets, and classic Forex markets, see for example Ferreira and Dionísio (2016); Kristoufek (2018a); Kumar and Kamaiah (2016). Recently, the topic has also gained increased interest in the crypto-community and in particular for Bitcoin markets.

Urquhart (2016) analyses the weak-form efficiency of Bitcoin by verifying pertinence of a random-walk hypothesis. While his results reject efficiency on the whole sample, from 2010 to 2016, Bitcoin returns seem to be random from 2013 onward. In a subsequent study, Nadarajah and Chu (2017) use the same daily Bitcoin price data and apply a power transformation to the returns. The authors then repeat Urquhart's analysis and find that the so transformed Bitcoin prices are efficient. A third extension of these results can be found in Bariviera (2017); Bariviera et al. (2017) who discover a regime-switch from inefficient to efficient prices in late-2013. More recently, Brauneis and Mestel (2018) extend the study of weak-form efficiency to 73 cryptocurrencies and link efficiency to market liquidity and size of the respective cryptos. Their results show that among the cryptos analyzed Bitcoin is the "least predictable" or "most efficient", respectively. In addition, results suggest that market liquidity as well as market capitalization positively affect efficiency. Along the same lines, Sensoy (2018) use intraday data on Bitcoin prices expressed in USD and EUR and find that both markets have become more efficient since beginning of 2016, that the USD market is more efficient than the EUR market during the period observed, informational efficiency decreases for higher data frequency, and efficiency is positively (negatively) affected by market liquidity (volatility).

A parallel stream of work approaches informational efficiency by testing weekday effects on Bitcoin prices which, again, would contradict the EMH. In a first study, Kurihara and Fukushima (2017) indeed find statistically significant weekday effects and conclude that Bitcoin markets are not efficient. On the other hand, separating the observation period into two parts they also 
find proof for Bitcoin markets transitioning to efficient markets. In an extended analysis Caporale and Plastun (2017), the authors find similar results for Bitcoin but do not find consistent return anomalies for other crypto-currencies.

A third workstream focuses on an analysis of long-range memory in Bitcoin prices which, if existing, would point to rejection of the weak-form EMH. For example, Tiwari et al. (2018) use daily prices over the period of July 2010 to June 2017 and various estimators including an efficiency index constructed from certain estimators first proposed in Kristoufek and Vosvrda (2013). Tiwari concludes from his analysis that Bitcoin markets are efficient except for some time periods and, hence, confirms previous results and, in particular, Urquart's results. However, while Urquart suggested that Bitcoin markets have become more efficient the efficiency index computed over time in Tiwari seems to indicate decreasing efficiency towards the end of the sample. Kristoufek (2018b) use the same efficiency index and extend the analysis to Bitcoin prices expressed in Chinese Yuan. Results do not confirm earlier findings of increasing efficiency but suggest that Bitcoin markets remain largely inefficient throughout the observation period.

Similar results are presented in Jiang et al. (2018) who apply a different efficiency index going back to Sensoy and Hacihasanoglu (2014), AlvarezRamirez et al. (2018) who use the detrended fluctuation analysis over sliding time windows, Al-Yahyaee et al. (2018) for a comparative analysis of efficiency of the Bitcoin, Stock, Gold, and Currency markets using the multi fractal detrended fluctuation analysis (MF-DFA), and Lahmiri et al. (2018) who explore presence of long-range memory in price volatility series for seven Bitcoin markets. Further, in a multidimensional analysis Cheah et al. (2018) study dynamic interdependence of Bitcoin prices across various markets in a fractionally cointegrated VAR framework. Long-memory is found in both the markets individually and the system of markets confirming informational inefficiency of Bitcoin.

Another workstream has focused on the semi-strong form of efficiency. For instance, Bartos (2015) analyzes the semi-strong form of the EMH for Bitcoin markets by correlating price to news events. Therefore, Bartos uses daily Bitcoin prices expressed in USD and aggregated across several exchanges over the period of March 2013 to July 2014. Further, two variables one representing daily internet user search queries for the word "Bitcoin" and the second expressing positive and negative news based on press articles ( 8 positive and 6 negative articles) about Bitcoin is used to measure availability of (new) public information on Bitcoin. Bartos' results show that positive as well as negative news announcements have a statistically significant explanatory power for the price of Bitcoin or, in other words, the Bitcoin price reflects such publicly available information as postulated by the semi-strong EMH. Hence, Bartos concludes that Bitcoin markets are, indeed, efficient. Using an extended data set covering the period of September 2011 to December 2017 Vidal-Tomás and Ibañez (2018) also tests for the semi-strong efficiency of Bitcoin. Their data includes 50 events (28 negative events and 22 positive) expressing monetary policy and Bitcoin related regulation announcements across the globe. The re- 
sults of this study indicate that Bitcoin does not respond to monetary policy news which, according to the authors, confirms that Bitcoin is detached from the real economy. On the other hand, with respect to Bitcoin-related events the study finds that the Bitcoin price only partially reflects new negative information and, hence, the authors do not confirm Bartos' results but find that Bitcoin markets are not semi-strong form efficient.

The results reviewed above are inconclusive as to whether Bitcoin markets are weak-form or semi-strong form efficient or not. Generally, authors observe times during which markets are efficient and for other periods they are not. In fact, Khuntia and Pattanayak (2018) confirms this patterns of efficiency and argues that these findings supports the adaptive market hypothesis (AMH) introdcued in Lo (2004). In analogy to evolutionary principles, the AMH assumes that markets evolve and adapt and that, as a consequence, market efficiency varies in degree along time.

We here propose to challenge the EMH for Bitcoin by proposing simple univariate trading strategies, based on straightforward signal extraction and forecasting principles, whose performances appear remarkably consistent and resilient over time, beyond the singular break-down of the currency in early 2017.

\section{The Data: BTC/USD-Market}

We use historical data of the Bitcoin (BTC) price expressed in USD, i.e. the BTC/USD exchange rate, from the Bitstamp exchange. As shown by Hileman and Rauchs (2017) Bitstamp is one of the top Bitcoin exchanges accounting for about $7 \%$ of the Bitcoin trading volume in the period of February to March 2017 and more than $60 \%$ of the Bitcoin trading volume was settled in USD by March 2017. Therefore, we believe that this data provides a good representation for Bitcoin market activity.

We access the Bitstamp Bitcoin price data through the Quandl AP ${ }^{1}$ Thereby, we are able to collect daily Last, Bid, Ask, and Volume data for the period of April 15, 2014 to Januar 10, 2019. Note that, since Bitstamp is trading on 24 hours a day and 7 days a week these prices refer to the last observed trade price, the current highest Bid and Ask offers, and the last 24 hours trading volume which are sourced by Quandl at 7pm EST every day. Further, it should be noted that the start of our data sample is different from other samples used in previous work. For instance, Urquhart (2016) uses Bitcoin price data starting at August 10, 2010 accessed through www.bitcoinaverage.com. This data, however, represents a Bitcoin price index constructed as the volumeweighted average Bitcoin price from all available Bitcoin exchanges worldwide. Therefore, while the latter source provides a wider time window our sample is

\footnotetext{
1 Quandl is a general data market place that collects and makes available public as well as commercial data sets through a unified API. For more information, visit https://quandl. com
} 
reproducible as it originates in a single market place and can be straightforwardly accessed.

Fig. 1 gives an overview of the observed Bid price (price), the logarithm of the Bid price (log-price), first differences of log-prices (log-returns) and the logarithm of 24-hours trading volume (log-volume) series. As shown in the figure, the price of 1 Bitcoin moved below USD 1,000 from the start of the observed period until beginning of 2017 and skyrocketed up to almost USD 20,000 on December 17 in the same year. In the last period, the price then steadily decreased again to a level of about USD 4,000 at the end of the observed period. The dynamics are impressive: the mean yearly return of a passive buy-and-hold strategy would amount to $150 \%$ and absolute daily returns in exceedance of the $10 \%$ mark are observed at 59 days which amounts to a probability of $3.45 \%$. Since both the drift as well as the volatility of the series are unusually large, when compared to classic assets, we compute the Sharpe ratio, which is a measure that balances drift and volatility aspects. For the Bitcoin data, the annualized Sharpe ratio is 0.59 in the considered time span, which is a commendable number for a passive strategy. At the same time, the 24-hours trading volume seems to exhibit some cyclical pattern with the year 2016 showing low trading volume, year 2017 a steady increase in volume building up to the almost USD 20,000 best-price mark at the end of 2017 and trading activity comparable to pre-2016 levels, while the trading activity decreased again sharply after that and continuously throughout most of the year 2018, increasing again for a short period at the end of 2018.

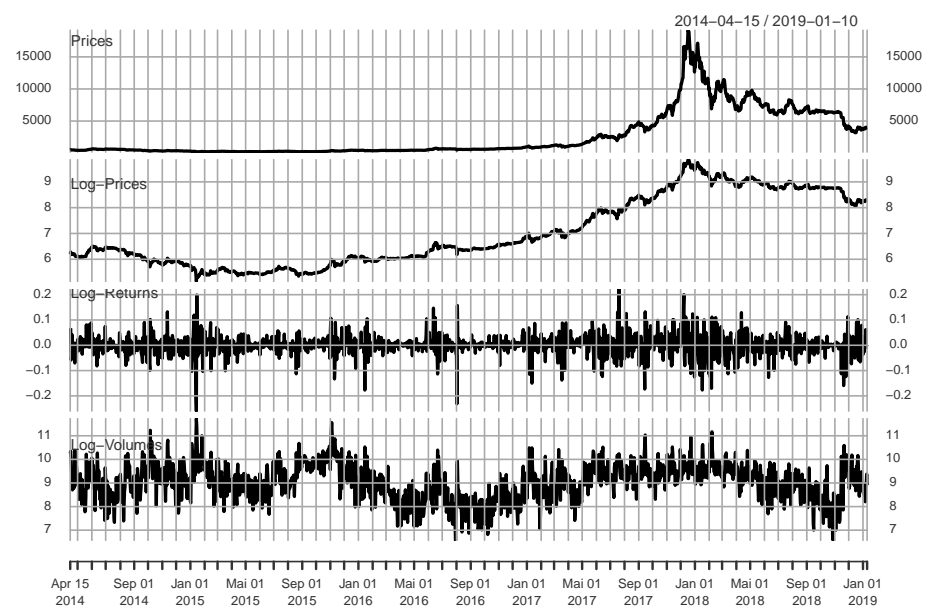

Fig. 1 Historical bid price (top panel), log-price (second panel), log-returns (third panel), and log-volumes (bottom panel) for Bitcoin (in USD) sourced from the Bitstamp exchange. 


\section{Unfolding Serial Dependence}

In the light of our research question, we now analyse log-returns $r_{t}=\log \left(\frac{P_{t}}{P_{t-1}}\right)$ of the Bitcoin price $P_{t}$ and check pertinence of the white noise assumption or, equivalently, of the random-walk hypothesis for the (log-)prices. The autocorrelation function (acf) of the log-returns is displayed in Fig. 2, left panel. Further, accounting for the observed volatility-clustering we also fit a GARCHmodel to the returns (results are computed with the R-package fGARCH). Model identification and diagnostic tests suggest pertinence of an MA(6)$\operatorname{GARCH}(1,1)$ specification

$$
\begin{aligned}
r_{t}= & \epsilon_{t}+0.035 \epsilon_{t-1}+0.016 \epsilon_{t-2} \\
& +0.048 \epsilon_{t-3}+-0.016 \epsilon_{t-4}+0.029 \epsilon_{t-5}+0.097 \epsilon_{t-6} \\
\sigma_{t}^{2}= & 0.00006+0.794 \sigma_{t-1}^{2}+0.184 \epsilon_{t-1}^{2}
\end{aligned}
$$

The resulting standardized model-residuals $u_{t}:=\epsilon_{t} / \sigma_{t}$ as well as the squared residuals $u_{t}^{2}$ pass all diagnostic checks, in particular the Ljung-Box statistics at lags 5, 10 and 15 routinely calculated by the fGARCH-package are $0.47,0.53,0.66$ for $u_{t}$ (and similarly for $u_{t}^{2}$ ).
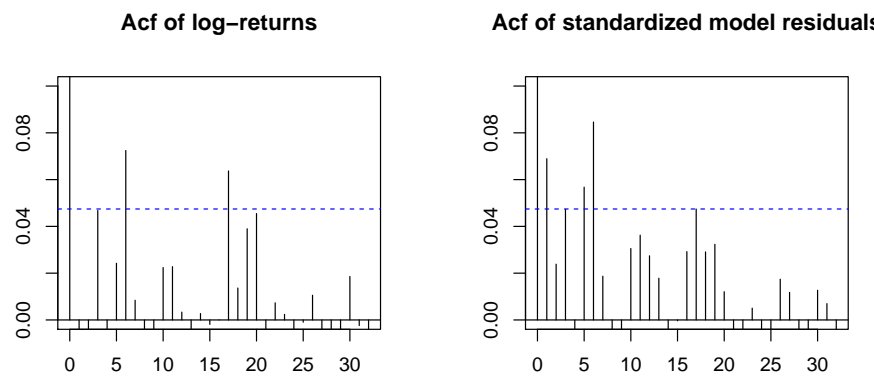

Fig. 2 Autocorrelation function (Acf) up to lag 30 for log-returns (left panel) and of standardized model residuals (right panel) for Bitcoin.

The MA(6) coefficient is largest, as expected, and strongly significant with a p-value of 0.00019 , thus confirming the previous analysis of the autocorrelation structure. Furthermore, the autocorrelation structure of the standardized model residuals in the right panel of Fig. 2 stretches at least up to lag 30. The empirical significance level of the Ljung Box statistic of the standardized model residuals at lag six amounts to 0.00003 and thus confirms strong significance of the disclosed dependency structure. We note, also, that the peak of the autocorrelation at lag 6 seems persistent across time, so for example this number amounts to 0.17 when using data of the year 2014, only, and it is statistically significant despite the relatively modest sample size. 
We infer that the log-returns of the series are subject to systematic and significant positive autocorrelation, which points towards a possible marketinefficiency that could be exploited by suitable trading strategies. The question whether the observed dependency structure is sufficient for balancing trading costs and to outperform the already impressive passive buy-and-hold strategy under these circumstances will be analyzed below.

\section{Systematic Trading Strategies}

The above data analysis has revealed dependency-structures which contradict the (weak form) EMH and which could be exploited by suitable forecast techniques. We propose three variants: moving-average filters, frequently found in momentum trading strategies, ARMA time series models and neural nets. Whereas the former two can exploit the statistically significant linear dependency-structure of the log-returns, the latter can account for possible non-linearity in the data, additionally.

\subsection{Trading Based on Signal Extraction Principles}

Momentum trading is a technique in which traders buy or sell an asset according to the direction of its trend. The trend $y_{L}, y_{L+1}, \ldots$ can be estimated from the data $x_{1}, x_{2}, \ldots$ by aggregation of the most recent observations

$$
y_{t}=\sum_{k=0}^{L-1} b_{t k} x_{t-k}
$$

where $b_{t k}$ are the coefficients of a one-sided (causal) filter, which may depend on time $t$, and $L<T$ is the filter-length. In some cases it is meaningful to consider normalized filters whose coefficients add to one: $\sum_{k} b_{t k}=1$. The simplest time invariant normalized smoothing filter is the equally-weighted moving average, or $\operatorname{EqMA}(L)$ in short-notation, with coefficients $b_{k}:=\frac{1}{L}$, $k=0, \ldots, L-1$, see for example Faber (2007). As an alternative, the wellknown normalized exponential-smoothing filter $b_{k}=\frac{1-\alpha}{1-\alpha^{L}} \alpha^{k}, k=0, \ldots, L-1$, abbreviated as $\operatorname{EMA}(\alpha)$, emphasizes the most recent observations more heavily than the remote past which is generally felt as a desirable property. Some classic momentum strategies rely on (log-) price differences $y_{t}=x_{t}-x_{t-\text { lag }}$, abbreviated as Diff(lag), where lag is typically a calendar-based time span like a year, a quarter, a month or a week, see for example Saravelos et al. (2018). In this case $b_{0}=1, b_{\text {lag }}=-1$ and $b_{k}=0$ for all other $k$.

Given the different characteristics of these filters, we are faced with the problem of selecting a pertinent design and we propose to base our decision upon signal extraction principles. For that purpose consider the following simple local linear level model

$$
\begin{aligned}
& x_{t}=\mu_{t}+\nu_{t} \\
& \mu_{t}=\mu_{t-1}+\epsilon_{t}
\end{aligned}
$$


with noise variances $\operatorname{Var}\left(\epsilon_{t}\right)=\sigma_{\epsilon}^{2}$ and $\operatorname{Var}\left(\nu_{t}\right)=\sigma_{\nu}^{2}$. Here, the user is interested in estimating the level $\mu_{t}$ from a sample $x_{1}, x_{2}, \ldots$ of the data, see e.g. Harvey (1990). If $\sigma_{\epsilon}^{2}>0$ then under classic model assumptions the optimal (in a meansquare sense) one-sided filter for estimation $\mu_{T}$ at the sample end $t=T$ is an EMA whose $\alpha$ depends on the signal-to-noise ratio (SNR) $\sigma_{\epsilon}^{2} / \sigma_{\nu}^{2}$. The larger the SNR the lesser the smoothing by the filter: for $\sigma_{\epsilon}^{2} / \sigma_{\nu}^{2} \rightarrow \infty$ an identity filter is obtained (because, trivially, the above system simplifies to $x_{t}=\mu_{t}$ in this case); for $\sigma_{\epsilon}^{2} / \sigma_{\nu}^{2}=0$ an EqMA-filter is obtained (because in this case $x_{t}=\mu+\nu_{t}$ is white noise plus a constant $\left.\mu\right)$; an ordinary EMA is obtained in-between these two extreme cases.

This model-based perspective justifies heavy smoothing in the case of noisy data, which would suggest applications to first differences or log-returns of the original prices. In such a case $\mu_{t}$ would correspond to the local drift of the price so that a simple trading strategy could be based upon buying or selling an asset depending on the sign of the optimal estimate $\hat{\mu}_{T}$ of $\mu_{T}$ at the sample end $t=T$. Applying the above local linear level model to the log-returns of Bitcoin, the signal to noise ratio obtains as $\sigma_{\epsilon}^{2} / \sigma_{\nu}^{2}=0.000028$ which, being close to zero, suggests pertinence of an equally-weighted filter design.

We infer that the usage of EqMAs, as applied to log-returns of Bitcoin data, could be justified based on signal extraction principles and use this design for our momentum trading strategy.

\subsection{Trading Based on Classic Time Series Models}

The previous EqMA-designs apply equal weights to current and past observations. A potentially more refined weighting scheme, at least in terms of forecasting, could be obtained by relying on the MA(6)-model (1) proposed in the previous section. The model can be inverted into its infinite autoregressive representation

$$
x_{t}=\sum_{k=1}^{\infty} a_{k} x_{t-k}+\epsilon_{t}
$$

from which the forecast weights $a_{k}$, assigned to past observations $x_{t-k}$, can be derived, see Fig. 3 . 


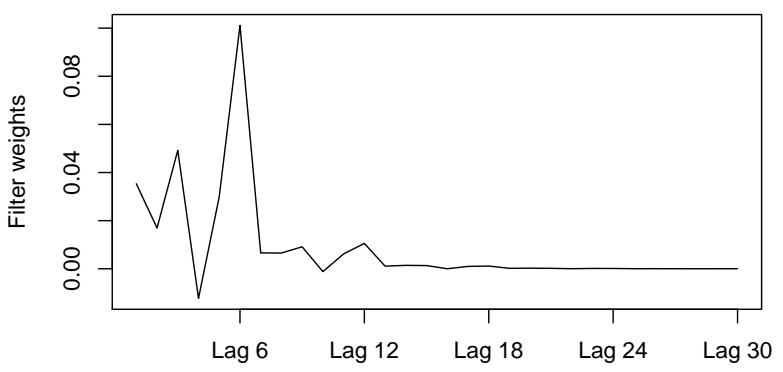

Fig. 3 Forecast filter weights of the selected MA(6) model.

The weight at lag 6 , with respect to the future observation $x_{t+1}$, is maximal as expected from the autocorrelation structure of the data.

\subsection{Trading Based on Neural Nets}

In order to expand our analysis further, we here propose to consider non-linear neural nets, interpreted in terms of non-linear forecast rules: outperformance of the two previous linear approaches by the neural nets would suggest evidence of 'non-linear' data-features. For simplicity of exposition we here restrict the analysis to feedforward nets with two hidden layers of dimensions six and three, see Fig. 4 .

Each node in the hidden layers corresponds to a neuron with a sigmoidactivation function

$$
\sigma\left(x_{1}, x_{2}, \ldots x_{n}\right)=\frac{1}{1+\exp \left(-b-\sum_{k=1}^{n} w_{k} x_{k}\right)}
$$

Specifically, at each time point $t$, the neurons $\sigma_{i 1}, i=1, \ldots, 6$, in the first hidden layer receive data $r_{t}, r_{t-1}, \ldots, r_{t-5}$ : the first six lags of the log-returns. Their outputs

$$
\sigma_{i 1}\left(r_{t}, \ldots, r_{t-5}\right)=\frac{1}{1+\exp \left(-b_{i 1}-\sum_{k=1}^{6} w_{k i 1} r_{t+1-k}\right)}
$$

for $i=1, \ldots, 6$ are fed to the neurons $\sigma_{j 2}, j=1,2,3$, in the second hidden layer, whose outputs are then fed to the single output neuron $\sigma_{\text {out }}$. The unknown parameters $b_{i j}$ (biases) and $w_{k i j}$ (weights) are displayed in Fig. 4. they are obtained by fitting (results are computed with the R-package neuralnet) the 
outputs $\sigma_{\text {out }}(t)$ of the net to the future returns $r_{t+1}$, relying on the classic mean-square error norm

$$
\sum_{t}\left(r_{t+1}-\sigma_{o u t}(t)\right)^{2} \rightarrow \min _{b_{i j}, w_{k i j}}
$$

The decision for the above net-configuration is based on our data-analysis (input-layer accounts for the first six lags of the data) as well as on a suitable compromise between flexibility and simplicity requirements (classic meansquare loss function as well as traditional sigmoid activation function): the results obtained by the above structure are representative for a fairly broad range of alternative net specifications or software implementations.

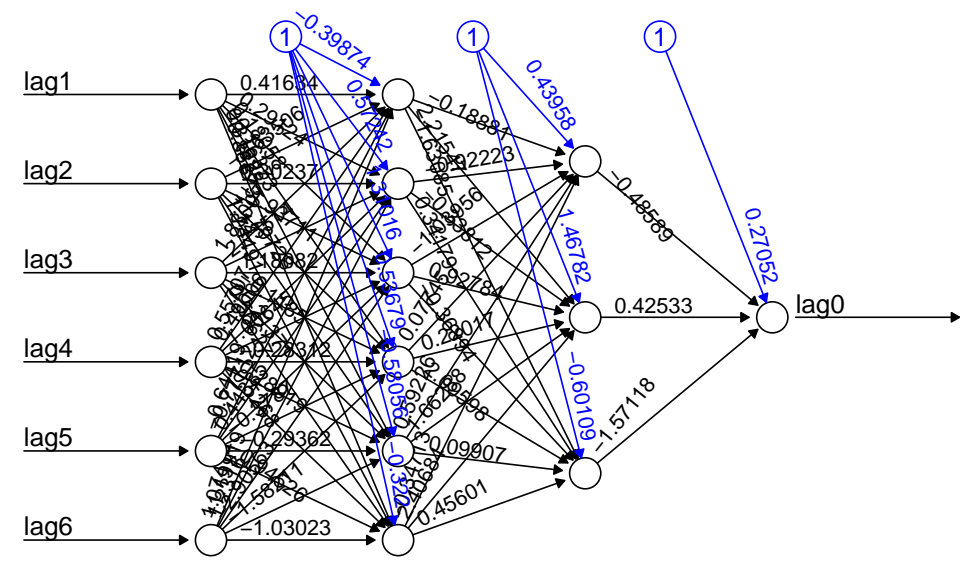

Fig. 4 Feedforward net with two hidden-layers applied. The 6 dimensions of the first hidden layer correspond to the first six lags of the Bitcoin log-returns.

As we have discussed above, under the EMH none of the above trading strategies could be persistently profitable and in fact they should all lead to systematic losses when accounting for the bid-ask spread at the corresponding trading time points. We will now challenge these claims, equipped with linear and non-linear filter-techniques.

\section{Empirical Results}

\subsection{Momentum-Strategy}

The previous analysis of prices, log-prices, and log-returns in section 3 has yielded some first interesting insights into the characteristics of Bitcoin. In particular, the autocorrelation function in Fig. 2 shows evidence of significant 
serial dependence in the log-returns up to lag 6 . Therefore, we propose to apply a simple $\operatorname{EqMA}(6)$-filter

$$
y_{t}=\frac{1}{6} \sum_{k=0}^{5} r_{t-k}
$$

to the log-returns $r_{t}, t=1,2, \ldots$ of Bitcoin and we adopt the strategy of buying or selling Bitcoin according to $y_{t}$ crossing the zero-line from below or from above.

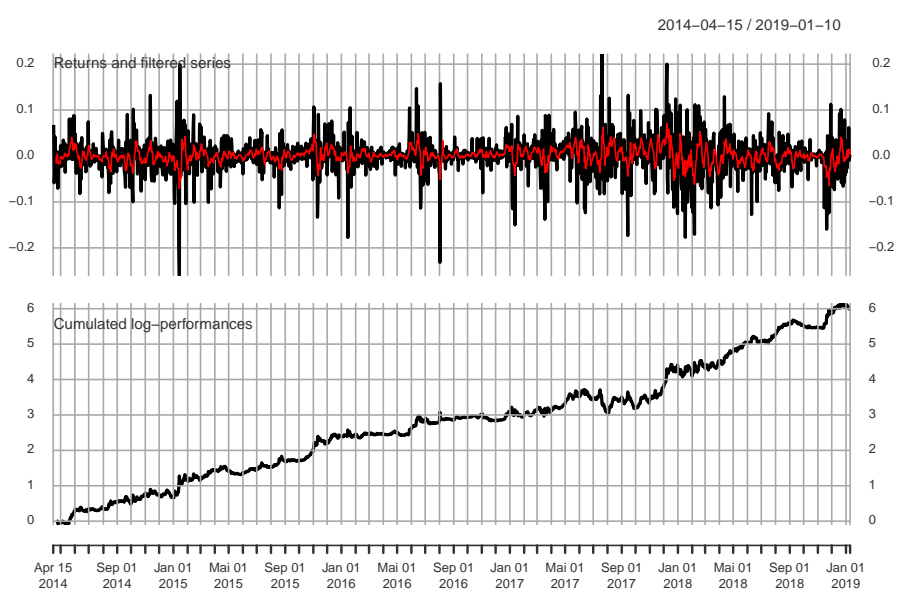

Fig. 5 Top panel: Log-returns (black) and filtered series (red). Bottom panel: cumulated (log-)performances of the momentum strategy based on EqMA(6) for Bitcoin.

Fig. 5 shows the original log-returns (black) and trading signal $y_{t}$ (red) in the top panel and the cumulated performances of our momentum trading strategy in the bottom panel. Note that trading costs are ignored here for simplicity (see below for corresponding results). An evaluation of our strategy and a comparison with buy-and-hold is provided in Table 1 ; the former outperforms the latter across the board. The empirical significance level of a test

\begin{tabular}{rrr}
\hline & Buy and hold & EqMA(6) \\
\hline Hit rate & 0.541 & 0.614 \\
Max draw down & 0.886 & 0.545 \\
Annualized Sharpe & 0.593 & 1.717 \\
\hline
\end{tabular}

Table 1 Performance measures: a comparison of the buy-and-hold strategy and EqMA(6) momentum strategy for Bitcoin.

for whether the two Sharpe ratios differ significantly amounts to $6.02 \%$ and misses the $5 \%$ mark only marginally (and similarly for the hit-ratio). 
Continuing our performance analysis we compute in Fig.6 the yearly return of the strategy (yearly differences of the cumulated performances) over the entire period. First of all, our results show strictly positive returns over the entire period which itself is impressive. Further, the yearly returns seem to be fluctuating around a return of $400 \%$ p.a. until the first third of the observed period where they drop to a level of about $200 \%$ p.a. in the second third of the sample period. Interestingly, from then on the yearly returns steadily lift-up to hit above $1000 \%$ p.a. towards the end of the sample period and settle on a level of about $600 \%$. This points to the fact that the Bitcoin market inefficiency becomes more accentuated in the last period of our sample, which contrasts with previous findings in Urquhart (2016); Kurihara and Fukushima (2017); Bariviera (2017) stating increased efficiency after around 2013 (though it is fair to mention that our data sample stretches two years further to the right than theirs).

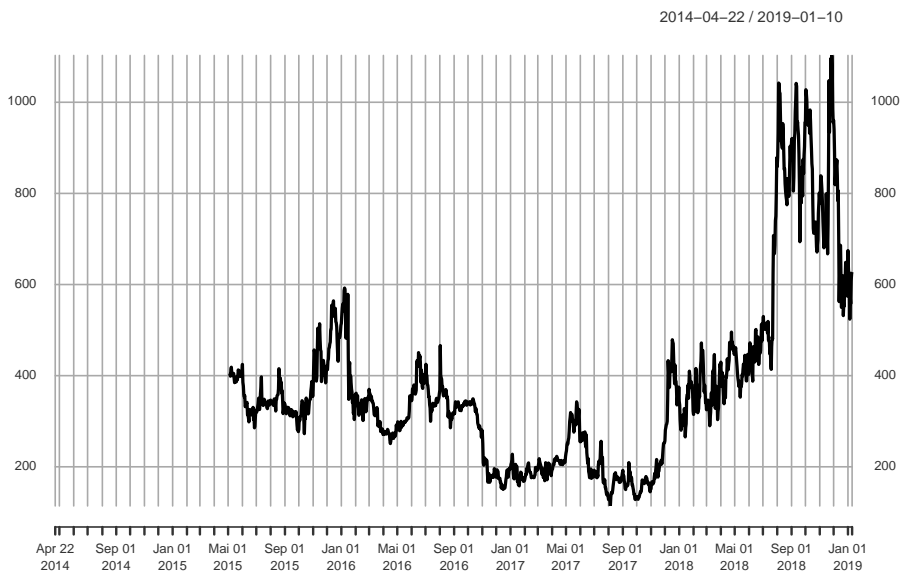

Fig. 6 Mean yearly return of the momentum strategy based on EqMA(6) for Bitcoin.

A test of the hypothesis that the drift of the resulting performance is larger than zerd ${ }^{2}$ leads to a value of the corresponding $t$-statistic of 3.7 or empirical significance level $0.011 \%$, respectively, thus providing additional evidence against the EMH. To conclude, we note that the above results may claim out-of-sample validity since the only freely determined parameter, namely the

\footnotetext{
2 The test-statistic is based on t-test $:=\overline{d_{t}} /\left(\sigma_{d_{t}} / \sqrt{T-L-1}\right)$ where $d_{t}, t=L+1, \ldots, T$ are first differences of the performance curve (which starts in $t=L$ where $L=6$ is the filterlength), $\overline{d_{t}}$ and $\sigma_{d_{t}}$ denote the arithmetic mean and the empirical standard deviation of $d_{t}$, so that $\left(\sigma_{d_{t}} / \sqrt{T-L-1}\right)$ is the standard deviation of the mean, assuming independence of $d_{t}$ (null hypothesis). We rely on the t.test-function in the R-package for deriving empirical significance levels of the statistic where we selected the one-sided test of the null-hypothesis of a vanishing drift against the alternative of a positive drift.
} 
filter-length, was obtained from a straightforward analysis of the autocorrelation function whose main feature, the peak at lag 6 , is pretty stable over time as shown in section 4

\subsection{Classic Time Series Models}

We here rely on the forecast-filter derived from the MA(6)-model (1). Specifically, we buy or sell the Bitcoin depending on the sign of the forecasts. Cumulated performances of the resulting strategy are displayed in Fig. 7 together with the buy-and-hold benchmark.

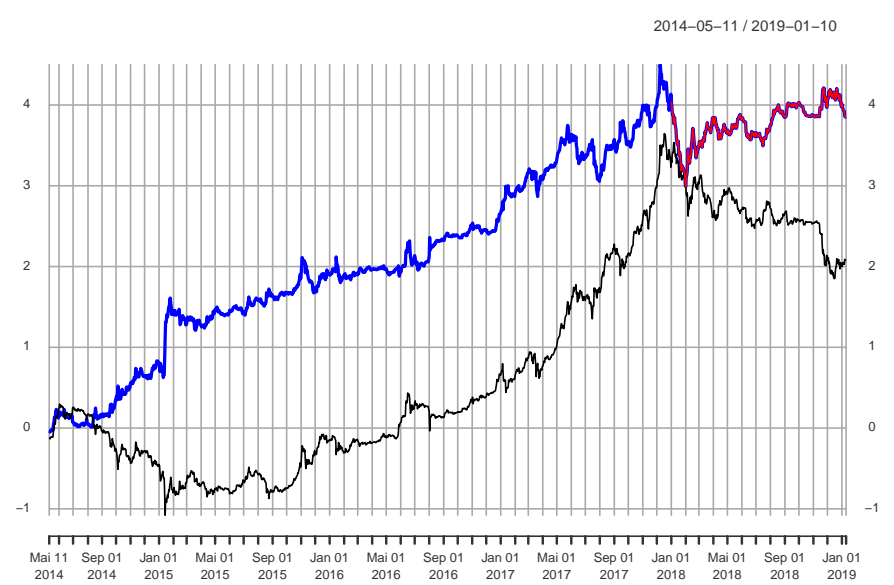

Fig. 7 Cumulated performances of the MA(6) forecast model strategy (blue=in-sample, red=out-of-sample) vs. the buy-and-hold strategy (black) for Bitcoin.

Except for a short contraction, coinciding with the draw-down of Bitcoin in early 2018, model-performances are fairly regular over the observed time span.

\begin{tabular}{rrr}
\hline & Buy and hold & MA(6) model \\
\hline Hit rate & 0.541 & 0.573 \\
Max draw down & 0.886 & 0.805 \\
Annualized Sharpe & 0.593 & 1.122 \\
\hline
\end{tabular}

Table 2 Performance measures: a comparison of the buy-and-hold strategy and MA(6) forecast model strategy for Bitcoin.

Performance measures of the resulting strategy are reported in Table 2 The time series model beats buy-and-hold on all accounts, but the extent is less marked than for the previous simpler EqMA(6) strategy. 


\subsection{Neural Nets}

The trading strategy applied in this section builds on a return forecast through a neural net time series model outlined in section 5.3. Analogously to the previous strategy, which used a classic time series model for return forecasting, the sign of our Bitcoin return forecast again indicates whether we buy or sell.

In contrast to the previous linear approaches, fitting of unknown parameters is generally more challenging for neural nets because the numerical optimization tends being trapped into local minima. Therefore, parameter estimates ordinarily depend upon suitable initial values for these parameters. In this context it is common to rely on random initializations of biases and weights: each random seed thus generates a new (random) net whose parameters may differ substantially from realization to realization. In order to illustrate the extent of this problem on trading outcomes, we compare cumulated in-sample (left panel) and out-of-sample (right panel) performances of 100 random nets in Fig. 8
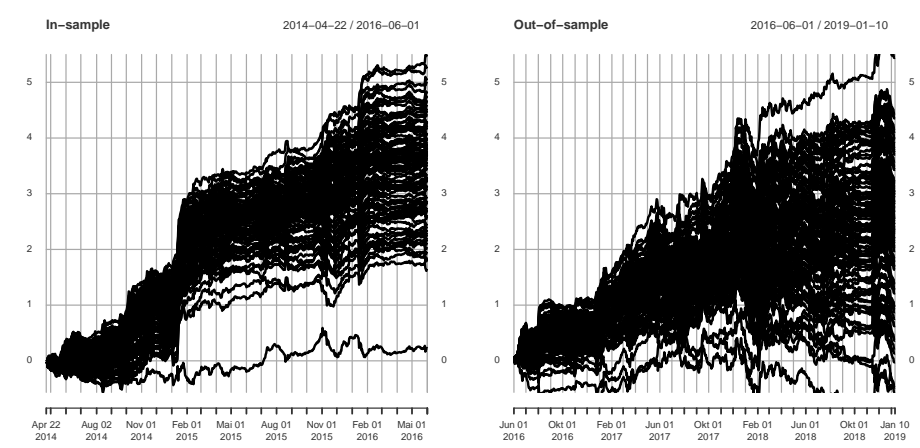

Fig. 8 Cumulated performances of 100 (random) nets applied to log-returns of Bitcoin: in-sample (left panel) and out-of-sample (right panel)

Training and validation samples were roughly split into $1 / 2$ and $1 / 2$ of the dataset so that the in-sample period ends in 2016-06-01. A quick glance at both graphs illustrates the effect of the random seed on trading performances: for example annualized Sharpe ratios vary in a range from 0.17 to 4.25 , in-sample, and in a range from -0.26 to 2.56 , out-of-sample. In-sample performances are overly optimistic due to overfitting, as expected. Interestingly, out-of-sample gains seem to be quite substantial, in the mean over all realizations, even after the breakdown of the Bitcoin in early 2018. The out-of-sample results in Fig. 8 reveal only 4 negative outcomes out of 100 at the sample end and all of them are close to flirting with the zero-line. At this stage of the analysis we may be interested in finding out if in-sample numbers (trading performances or fore- 
cast performances) are informative about future out-of-sample performances. Specifically, the correlation between in-sample and out-of-sample Sharpe ratios amounts to 0.56 suggesting that well- (bad-) performing designs will tend systematically to out- (under-) perform in the future, too. However, the correlation between in-sample forecast performances (parameter-fitting criterion) and out-of-sample Sharpe ratios, namely - 0.53 , dissipates doubts on this appreciation. Overcoming these conflicting evidences, we could rely on a simple ensemble average, the cross-sectional mean, of all performances as shown in Fig. 9: the comparison of the neural net (red line) and the linear MA(6) models (blue line) may help shedding some light on the empirical evidence of non-linearities in the Bitcoin returns.

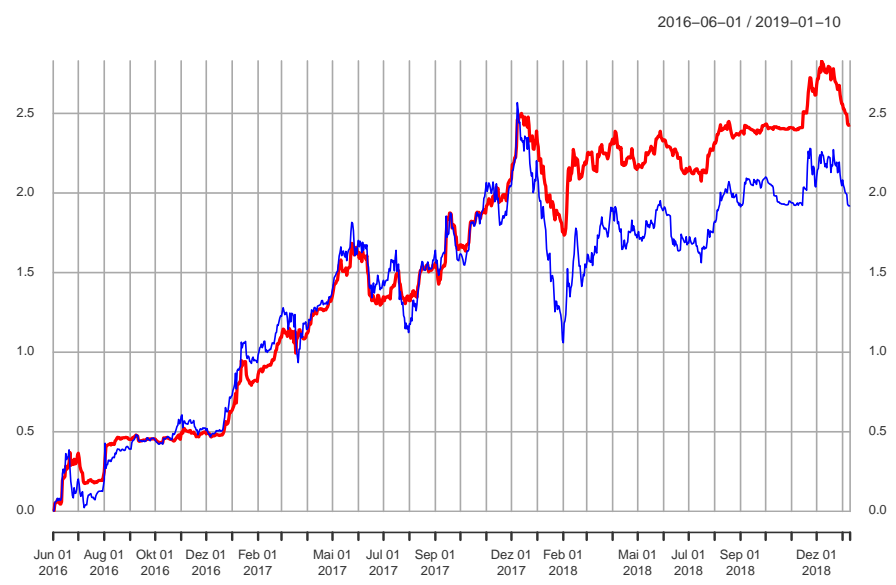

Fig. 9 Average cumulated out-of-sample performances (across 100 random nets) for the neural net forecast model (red) vs. MA(6) forecast model (blue) strategies for Bitcoin.

Indeed, a quick glance at both curves suggests fairly similar performances, except perhaps for the heavier draw-down of the classic model at the beginning of 2018. Performance numbers of all strategies for the out-of-sample span starting in 2016-06-01 are reported and compared in Table 3 note that these numbers may differ from the whole-sample numbers reported in the previous tables. The neural net's top-rankings are due, at least in part, to the ensemble averaging of the random nets and cannot be attributed entirely to 'non-linearity' of the time series, as discussed above. Buy-and-hold and the MA(6)-model are systematically outperformed by the other two strategies for the considered time span. The simple EqMA(6) challenges the neural net's ensemble averaging, suggesting that equal-weighting of the past 6 observations accounts in an effective and parsimonious way for the positive pattern of the autocorrelation function revealed in Fig.2. 


\begin{tabular}{|c|c|c|c|c|}
\hline & Buy and hold & $\operatorname{EqMA}(6)$ & MA(6) model & Neural net \\
\hline Hit rate & 0.562 & 0.624 & 0.590 & 0.679 \\
\hline Max draw down & 0.886 & 0.545 & 0.805 & 0.558 \\
\hline Annualized Sharpe & 0.941 & 1.581 & 0.899 & 1.639 \\
\hline
\end{tabular}

Table 3 Out-of-sample performance measures: a comparison of the buy-and-hold strategy, EqMA(6) momentum strategy, and $\mathrm{MA}(6)$ and neural ensemble average forecast model strategies for Bitcoin.

In order to verify significance of the above out-of-sample performances we compute the t-test for positive trading performances: the empirical significance levels are $0.56 \%$, for the $\operatorname{EqMA}(6), 7.49 \%$, for the MA(6)-model and $0.43 \%$, for the neural net (ensemble average). We may infer from Fig. 9 that the protracted down-turn in early 2018 is responsible for pushing the test-level of the linear MA(6)-model a smidge above the 5\%-mark.

\subsection{Trading Costs: Accounting for the Bid-Ask Spread}

To conclude, we briefly analyze the effects of trading-costs, by crossing the spread between bid and ask prices at each trade. We here restrict the analysis to EqMA-filters, since results are similar across all three approaches.

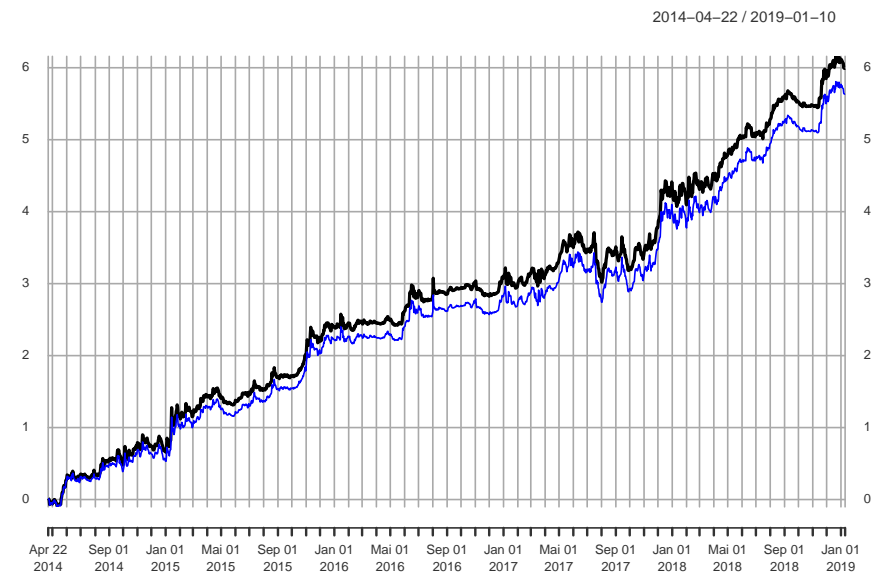

Fig. 10 Effect of trading costs (crossing the bid-ask spread) on performances of the momentum strategy based on $\operatorname{EqMA}(6)$ for Bitcoin.

Fig. 10 displays results for the above $\operatorname{EqMA}(6)$ : the (annualized) Sharpe ratio drops inconsequentially from 1.717 to 1.613 and the value of the above $t$-statistic drops insignificantly from 3.71 to 3.48 after accounting for the bid- 
ask spread. We may infer that the effect of the spread is negligible even for filters with relatively short holding periods, such as the EqMA(6).

\section{Conclusion}

Our aim was to check pertinence of the EMH for the Bitcoin. Data analysis suggested evidence for a violation of this assumption by revealing systematic significant positive serial correlation of the log-returns, which unfolded after accounting for volatility clustering. We then proposed three different trading strategies relying on simple equally-weighted moving average filters, derived from signal extraction principles, as well as on classic ARMA forecast models and non-linear neural nets. Our trading results confirmed the previous data-analysis, by highlighting a filter of length 6, or an EqMA(6), as the most effective momentum strategy. Its performances were strongly statistically significant and the course of the yearly return series suggested increasing market-inefficiency towards the sample end (Januar 10, 2019). Similar results were obtained for the two forecast approaches with a slight edge in favor of the ensemble average of random neural nets. A comparison of their trading performances out-of-sample suggested only modest departure from linearity, possibly during the draw-down of the Bitcoin at the beginning of 2018. Statistical significance could be established for all but the MA(6)-model which marginally missed the mark due to the aforementioned draw-down. Finally, we extended our performance analysis to the inclusion of trading costs by crossing the spread between bid and ask prices at each trade. Confirming the overall positive cumulative performances our results were only marginally affected by accounting for trading costs. In summary, our findings strongly reject the EMH for the Bitcoin market throughout the entire sample period and in particular in recent times. Departures from linearity appear marginal, possibly confined to the draw-down of Bitcoin in early 2018.

\section{References}

Al-Yahyaee K, Mensi W, Yoon S (2018) Efficiency, multifractality, and the long-memory property of the bitcoin market: A comparative analysis with stock, currency, and gold markets. Finance Research Letters

Alvarez-Ramirez J, Rodriguez E, Ibarra-Valdez C (2018) Long-range correlations and asymmetry in the bitcoin market. Physica A: Statistical Mechanics and its Applications 492:948 - 955

Bariviera AF (2017) The inefficiency of bitcoin revisited: A dynamic approach. Economics Letters 161:1 - 4

Bariviera AF, Basgall MJ, Hasperué W, Naiouf M (2017) Some stylized facts of the bitcoin market. Physica A: Statistical Mechanics and its Applications 484:82-90

Bartos J (2015) Does Bitcoin follow the hypothesis of efficient market? International Journal of Economic Sciences 4(2):10-23 
Baur DG, Dimpfl T, Kuck K (2018) Bitcoin, gold and the us dollar - a replication and extension. Finance Research Letters 25:103 - 110

Brauneis A, Mestel R (2018) Price discovery of cryptocurrencies: Bitcoin and beyond. Economics Letters 165:58 - 61

Brière M, Oosterlinck K, Szafarz A (2015) Virtual currency, tangible return: Portfolio diversification with bitcoin. Journal of Asset Management 16(6):365-373

Caporale GM, Plastun A (2017) The Day of the Week Effect in the Crypto Currency Market. Discussion Papers of DIW Berlin 1694, DIW Berlin, German Institute for Economic Research

Carrick J (2016) Bitcoin as a complement to emerging market currencies. Emerging Markets Finance and Trade 52(10):2321-2334

Chan S, Chu J, Nadarajah S, Osterrieder J (2017) A statistical analysis of cryptocurrencies. Journal of Risk and Financial Management 10:12

Cheah ET, Mishra T, Parhi M, Zhang Z (2018) Long memory interdependency and inefficiency in bitcoin markets. Economics Letters 167:18 - 25

Chuen L, LEE D, Guo L, Wang Y (2017) Cryptocurrency: A new investment opportunity? The Journal of Alternative Investments 20(3):16-40

Dyhrberg AH (2016) Bitcoin, gold and the dollar - a garch volatility analysis. Finance Research Letters 16:85 - 92

Faber M (2007) A quantitative approach to tactical asset allocation (february 2013). Journal of Wealth Management

Fama EF (1970) Efficient capital markets: A review of theory and empirical work. The Journal of Finance 25(2):383-417

Ferreira P, Dionísio A (2016) How long is the memory of the us stock market? Physica A: Statistical Mechanics and its Applications 451:502 - 506

Harvey AC (1990) Forecasting, Structural Time Series Models and the Kalman Filter. Cambridge University Press

Hileman G, Rauchs M (2017) 2017 global cryptocurrency benchmarking study

Jiang Y, Nie H, Ruan W (2018) Time-varying long-term memory in bitcoin market. Finance Research Letters 25:280 - 284

Kasper J (2017) Evolution of bitcoin - volatility comparisons with least developed countries. Journal of Internet Banking and Commerce 22(03)

Khuntia S, Pattanayak J (2018) Adaptive market hypothesis and evolving predictability of bitcoin. Economics Letters 167:26 - 28

Kristoufek L (2018a) Are the crude oil markets really becoming more efficient over time? some new evidence. Energy Economics

Kristoufek L (2018b) On bitcoin markets (in)efficiency and its evolution. Physica A: Statistical Mechanics and its Applications 503:257 - 262

Kristoufek L, Vosvrda M (2013) Measuring capital market efficiency: Global and local correlations structure. Physica A: Statistical Mechanics and its Applications 392(1):184 - 193

Kumar AS, Kamaiah B (2016) Efficiency, non-linearity and chaos: evidences from brics foreign exchange markets. Theoretical and Applied Economics XXIII(1(606), Spring):103-118 
Kurihara Y, Fukushima A (2017) The market efficiency of bitcoin: A weekly anomaly perspective. Journal of Applied Finance \& Banking 7(3)

Lahmiri S, Bekiros S, Salvi A (2018) Long-range memory, distributional variation and randomness of bitcoin volatility. Chaos, Solitons \& Fractals 107:43 $-48$

Lo AW (2004) The adaptive markets hypothesis. The Journal of Portfolio Management 30(5):15-29

Loi H (2017) The liquidity of bitcoin. International Journal of Economics and Finance 10:13

Nadarajah S, Chu J (2017) On the inefficiency of bitcoin. Economics Letters 150:6 - 9

Osterrieder J (2017) The statistics of bitcoin and cryptocurrencies. Advances in Economics, Business and Management Research 26

Osterrieder J, Lorenz J (2017) A statistical risk assessment of bitcoin and its extreme tail behavior. Annals of Financial Economics 12(01)

Petukhina A, Trimborn S, Härdle WK, Elendner H (2018) Investing with cryptocurrencies - evaluating the potential of portfolio allocation strategies. IRTG 1792 Discussion Paper 2018-058, Humboldt-Universität zu Berlin, Germany

Saravelos G, Gopal S, Grover R, Natividade C, Harvey O, Anand V, Winkler R, Kalani G (2018) Alive and kicking: A guide to fx as an asset class. Deutsche Bank Research

Sensoy A (2018) The inefficiency of bitcoin revisited: A high-frequency analysis with alternative currencies. Finance Research Letters

Sensoy A, Hacihasanoglu E (2014) Time-varying long range dependence in energy futures markets. Energy Economics 46:318 - 327

Tiwari AK, Jana R, Das D, Roubaud D (2018) Informational efficiency of bitcoin - an extension. Economics Letters 163:106 - 109

Urquhart A (2016) The inefficiency of bitcoin. Economics Letters 148:80 - 82

Vidal-Tomás D, Ibañez A (2018) Semi-strong efficiency of bitcoin. Finance Research Letters 27:259 - 265 